\title{
Multiple Cracks Propagate Simultaneously in Polymer Liquids in Tension
}

\author{
Qian Huang, ${ }^{1}$ Nicolas J. Alvarez, ${ }^{2}$ Aamir Shabbir, ${ }^{1}$ and Ole Hassager ${ }^{1, *}$ \\ ${ }^{1}$ Department of Chemical and Biochemical Engineering, Technical University of Denmark, 2800 Kongens Lyngby, Denmark \\ ${ }^{2}$ Department of Chemical and Biological Engineering, Drexel University, Philadelphia, Pennsylvania 19104, USA
}

(Received 22 February 2016; revised manuscript received 18 April 2016; published 15 August 2016)

\begin{abstract}
Understanding the mechanism of fracture is essential for material and process design. While the initiation of fracture in brittle solids is generally associated with the preexistence of material imperfections, the mechanism for initiation of fracture in viscoelastic fluids, e.g., polymer melts and solutions, remains an open question. We use high speed imaging to visualize crack propagation in entangled polymer liquid filaments under tension. The images reveal the simultaneous propagation of multiple cracks. The critical stress and strain for the onset of crack propagation are found to be highly reproducible functions of the stretch rate, while the position of initiation is completely random. The reproducibility of conditions for fracture points to a mechanism for crack initiation that depends on the dynamic state of the material alone, while the crack profiles reveal the mechanism of energy dissipation during crack propagation.
\end{abstract}

DOI: 10.1103/PhysRevLett.117.087801

Understanding the conditions for rupture of polymer melts and solutions under extension is a problem of both industrial and fundamental interest. For example, in the production of polymer fibers, a filament of a polymer melt is subjected to fast extensional flow in order to align the polymer molecules as much as possible. However, at the same time, rupture of the melt filament must be avoided. Polymer melts and solutions are viscoelastic materials, meaning that they behave in a fluidlike way on slow time scales and in a solidlike way on fast time scales. As a result of extensive studies, a consensus has emerged that two types of rupture modes may be identified for polymer liquid filaments, a viscoelastic instability mode and an elastic fracture mode [1]. The viscoelastic instability is also called necking instability [2] or ductile failure [3]. The mode generally occurs on a time scale that allows visual observation. It is generally assumed that an initially axissymmetric filament retains axis symmetry during rupture of this mode [4]. In contrast, our understanding of elastic fracture in polymer liquids is less satisfactory. While there has been progress in the understanding of fracture in soft solids [5-9] and model viscoelastic fluids [10,11], there is currently no generally accepted theoretical framework for describing the initiation and propagation of cracks in entangled polymer melts and solutions. Part of the problem is that elastic fracture typically occurs on such a fast time scale as to make direct visual observations impossible. Thus, even the simple question of whether the elastic rupture mode in entangled polymer liquid filaments retains axis symmetry or occurs via an edge fracture is unresolved.

The classical framework for crack propagation and brittle fracture was provided by the Griffith theory [12]. Inherent in the Griffith framework is the preexistence of material imperfections in the form of microcracks. When the applied load exceeds a critical value corresponding to the propagation threshold for the largest of the microcracks, that crack begins to grow catastrophically, leading to fracture. Since the size of the largest imperfection varies from sample to sample, the fracture stress in brittle materials is seldom reproducible. For liquids such as polymer melts and solutions, surface tension eliminates surface imperfections such as microcracks. The Griffith condition is therefore not immediately applicable to polymer melts and solutions. For viscoelastic materials, Tabuteau et al. [10] applied the model by Pomeau [13] and suggested that thermally induced fluctuations resulting in weaker domains play the role of crack initiators. Tabuteau et al. $[10,11]$ also showed that the fracture stress is typically of the order of the elastic modulus for the tested viscoelastic fluids. A consequence of the Pomeau model is that fracture conditions for viscoelastic liquids are much more reproducible than they are for brittle solids.

The work described here is motivated by the need for observations under well-defined conditions, from which a theoretical framework for fracture in polymer melts and solutions could be developed. In this Letter we show that the elastic rupture mode in entangled polymer liquid filaments is an edge fracture mode that breaks axis symmetry. We also show that the fracture stress is orders of magnitude higher than the elastic modulus. Moreover, the conditions for initiation of fracture in polymer liquid filaments are so well defined that multiple cracks are initiated independently and propagate simultaneously. Analysis of the crack profiles suggests that conditions near the crack tip may be in the glassy regime.

We investigated fracture in liquid bridges formed from two model entangled polymer liquids stretched in uniaxial extensional flows at constant stretch rates at $120^{\circ} \mathrm{C}$ (about $30^{\circ} \mathrm{C}$ higher than their corresponding glass transition temperatures $T_{g}$ ). The two samples, PS-864k/4k-33 
$\left(T_{g}=92^{\circ} \mathrm{C}\right)$ and PS-864k $/ 4 \mathrm{k}-17\left(T_{g}=89^{\circ} \mathrm{C}\right)$, consist of a nearly monodisperse polystyrene (PS) of $864 \mathrm{~kg} / \mathrm{mole}$ diluted in a styrene oligomer of $4 \mathrm{~kg} / \mathrm{mole}$, with PS volume fraction of $33 \%$ and $17 \%$, respectively. The styrene oligomer is well below the entanglement molecular weight $\left(M_{e}=13.3 \mathrm{~kg} / \mathrm{mole}\right)$ and can be considered as a solvent $[14,15]$. Such systems may be modeled by the highly successful tube theory for monodisperse linear polymer melts and solutions [16]. In particular, their linear viscoelastic (LVE) properties can be described by the three tube model [16] parameters: the number of entanglements per chain $(Z)$, the number of Kuhn segments per entanglement $\left(N_{e}\right)$, and the relaxation time of one entanglement $\left(\tau_{e}\right)$ (see Supplemental Material [17] to calculate $Z$ and $N_{e}$ ). The LVE properties are obtained from small amplitude oscillatory shear measurements and the result of PS-864k/4k-33 is shown in Fig. 1 (see Fig. S1 in Supplemental Material [17] for PS-864k/4k-17 and a commercial PS melt). The storage modulus $G^{\prime}$ represents the elastic property of the sample, while the loss modulus $G^{\prime \prime}$ represents the viscous property (energy dissipation). The three crossover points are associated with three time constants: the relaxation time of the polymer chain (also called reptation time, $\tau_{\text {rep }}$ ), the relaxation time of one entanglement $\left(\tau_{e}\right)$, and the relaxation time of one Kuhn segment $\left(\tau_{0}\right)$ [19]. The values of the parameters are listed in the inset of Fig. 1; the final time constant, the Rouse time $\tau_{R}=Z^{2} \tau_{e}$, is a characteristic time for relaxation of polymer chain stretching. The polymer liquids have two characteristic moduli: A glassy modulus $G_{0}$ that is the same for both liquids here and an elastic modulus $G_{e}$ that is proportional to the entanglement density. The latter is about four times bigger for PS-864k/4k-33 than for PS-864k/4k-17.

The samples were stretched in uniaxial extensional flows using the VADER 1000, Rheo Filament ApS, a commercially available filament stretching rheometer [20]. The inset

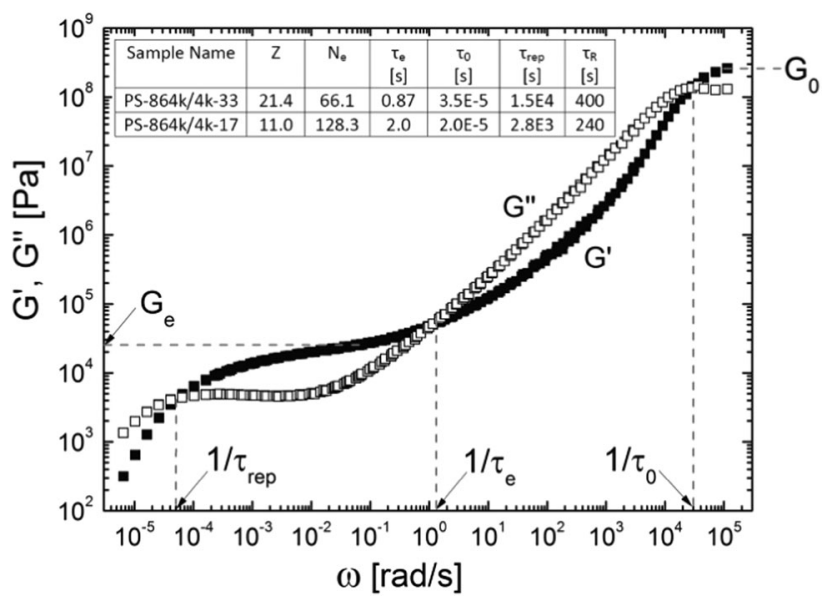

FIG. 1. Storage modulus $G^{\prime}$ and loss modulus $G^{\prime \prime}$ as a function of angular frequency $\omega$ for PS-864k/4k-33 at the reference temperature $120^{\circ} \mathrm{C}$. The inset lists the values of the tube model parameters for both PS-864k/4k-33 and PS-864k/4k-17 at $120^{\circ} \mathrm{C}$. of Fig. 2(a) shows an example of a filament (quenched) during stretching. Assuming incompressibility of the melts, the Hencky strain of the liquid in the midfilament plane is defined as $\varepsilon=-2 \ln \left(D / D_{0}\right)$, where $D_{0}$ is the initial diameter in the midfilament plane and $D$ is the diameter at time $t$. The diameters are measured by a laser micrometer during stretching and a control loop [21] is used to adjust the plate motion to ensure a constant value of the stretch rate defined as $\dot{\varepsilon}=d \varepsilon / d t$. Nondimensional stretch rates are given by the Weissenberg number $\mathrm{Wi}=\dot{\varepsilon} \tau_{R}$. All experiments leading to fracture have $\mathrm{Wi}>1$ meaning that the polymer chains are stretched [19].

We find the critical stress and strain for fracture to be highly reproducible functions of the stretch rates as indicated in. Fig. 2(a). For each stretch rate shown, there are two measurements presented, and in each case the fracture happens at the same stress and the same Hencky
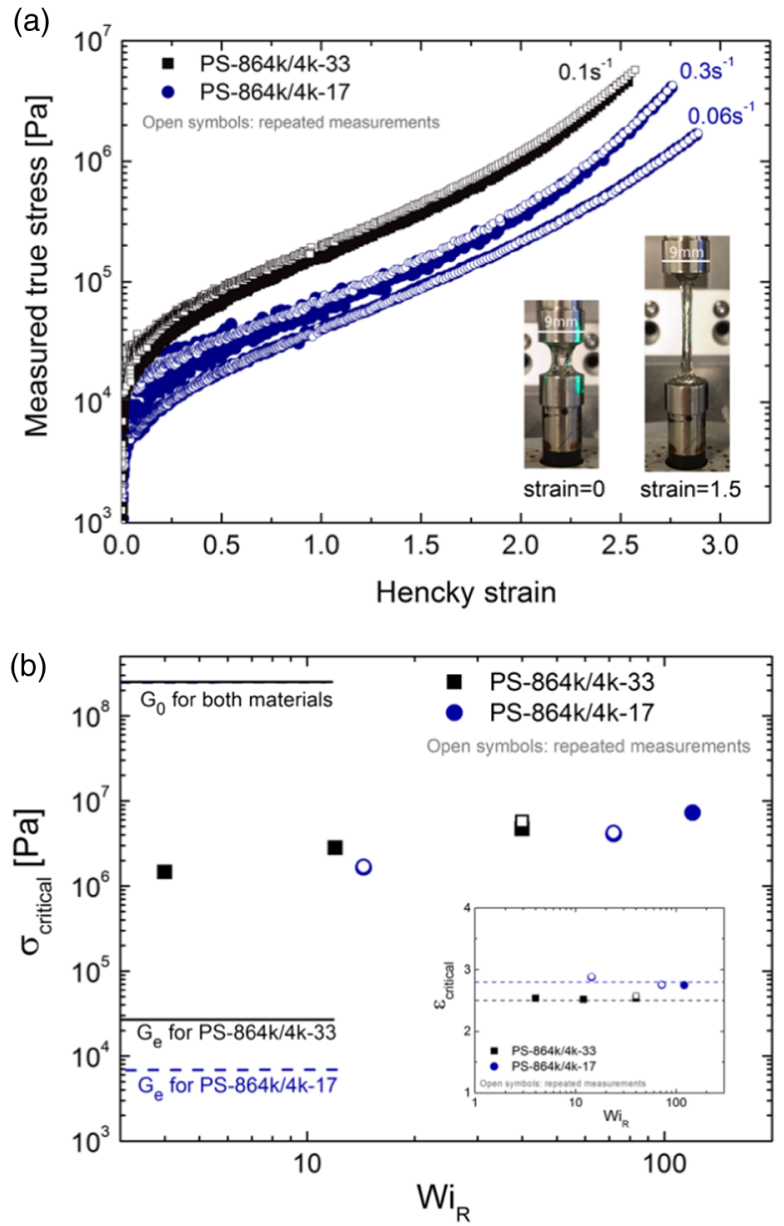

FIG. 2. Results of filament stretching measurements. (a) Measured stress as a function of Hencky strain at different stretch rates at $120^{\circ} \mathrm{C}$. For each rate the filament fractures at the end of the measurement. The inset shows the photos of one filament of PS-864k/4k-33 at Hencky strain 0 and 1.5, respectively. (b) Critical stress for fracture as a function of Weissenberg number Wi. The inset shows the corresponding critical strain at fracture as a function of Wi. 
strain (see also Fig. S2(a) in Supplemental Material [17]). In particular, for the stretch rate of $0.1 \mathrm{~s}^{-1}$, the two measurements were performed on samples with different initial sizes $\left(D_{0}=3.6\right.$ and $2.6 \mathrm{~mm}$, respectively), so there is no observable dependence on sample size. For the stretch rate of $0.06 \mathrm{~s}^{-1}$, after the experiment the two broken ends of the filament were pushed back together with an applied force. After waiting a time longer than the reptation time of the sample, the second measurement was performed and the reproducibility was almost perfect, in accordance with the liquid nature of the melts. It is well known that the rheological behavior of viscoelastic fluids follows the timetemperature superposition principle, whereby the internal time constants of a material may be adjusted by changing the temperature [22]. We use this principle to determine whether the stress limit is a function of the rate of stretching compared with the rate of relaxation of the sample only. For the two plots at $0.3 \mathrm{~s}^{-1}$ in Fig. 2(a), the first measurement was performed at $120^{\circ} \mathrm{C}$, while the second measurement was performed at $115^{\circ} \mathrm{C}$ at a lower stretch rate that corresponds to $0.3 \mathrm{~s}^{-1}$ at $120^{\circ} \mathrm{C}$. Importantly, the critical stress and strain are unchanged when the data measured at two different temperatures are shifted to the same reference temperature. This observation indicates that the "internal clock" of the melts plays an important role in the fracture process. Furthermore, Fig. 2(b) shows that with increasing stretch rate, the critical stress increases only slightly, and the critical strain is almost the same. The weak dependence on strain rate is in agreement with the thermally activated crack nucleation model for the fracture of viscoelastic fluids [23]. Moreover, while the entanglement density of PS-864k/4k-33 is almost four times that of PS-864k/ $4 \mathrm{k}-17$, they fracture at a similar critical stress. The value of the critical stress is between the corresponding elastic modulus $G_{e}$ and glassy modulus $G_{0}$ (see Fig. 1) of both liquids and is at least two orders of magnitude higher than $G_{e}$. This observation suggests that substantial chain stretch is involved in the fracture process, and that the relevant thermal fluctuations responsible for crack initiation happen on a shorter length and time scale than Rouse dynamics, i.e., shorter than entanglement dynamics.

While the dynamical conditions (stress and strain) are evidently highly reproducible, the position of the fracture is completely random [see Fig. S2(b) in Supplemental Material [17]]. High speed imaging of the fractures (Fig. 3; see also Fig. S3 in Supplemental Material [17]) reveals an additional phenomenon that further underpins the intriguing nature of fracture in viscoelastic liquids. Once conditions for fracture have been reached, more than one crack is initiated and multiple cracks propagate simultaneously. Figure 3 shows the captured cracks in PS-864k/4k-33 (see also movie S1 in Supplemental Material [17]). The entire process of crack propagation is rather short (about $200 \mathrm{~ms}$ ) compared with the time for stretching (about $26 \mathrm{~s}$ ). We let crack 1 refer to the main

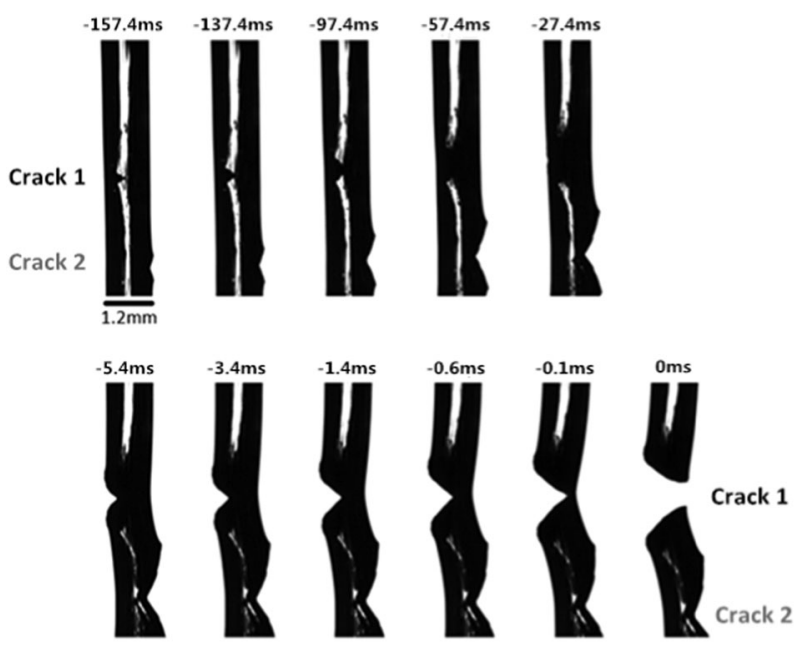

FIG. 3. Crack propagation in uniaxial extensional flow for PS- $864 \mathrm{k} / 4 \mathrm{k}-33$ stretched at $0.1 \mathrm{~s}^{-1}$ at $120^{\circ} \mathrm{C}$. The time $t=0$ is set at the frame where the filament breaks. The minus sign in the time for other frames means it happens before the fracture. The white line in the middle is light shining through the filament. The breaking of the line around $-150 \mathrm{~ms}$ reveals the existence of crack 1 , which is otherwise not visible before $-5.4 \mathrm{~ms}$ since it is on the front of the filament.

crack that leads to the complete fracture of the filament. The axial distance between observed multiple cracks along the filament amounts in some instances to more than four filament diameters [see Fig. S3(a) in Supplemental Material [17]]. This distance suggests that there is initially no stress field interaction between the propagating cracks, and that the initiation of crack 2 is independent of the existence of crack 1. As crack 1 grows, the stress near that crack continually increases due to the reduction in area. When crack 1 is roughly one third through the filament, it accelerates significantly as shown in Fig. 4(c), which shows the crack length $L$ as a function of time measured from image analysis of frames in Fig. 3. The velocities of crack propagation obtained from the slope of the plot are $2 \mathrm{~mm} / \mathrm{s}$ in the slow process and $70 \mathrm{~mm} / \mathrm{s}$ in the faster process, respectively. Once crack 1 reaches the point of filament failure the stress is relieved and all other cracks close up upon removal of the driving force. The crack propagation velocities may be compared with the velocity for transmission of a shear wave in a linear viscoelastic liquid [22]. This velocity is given by $v_{s}=\left(G^{*} / \rho\right)^{1 / 2} f$, where $\rho$ is the density, $G^{*}=\left(G^{\prime 2}+G^{\prime \prime 2}\right)^{1 / 2}$ is the absolute value of the complex modulus, $f=\left[2\left(x-x^{1 / 2}\right)\right]^{1 / 2} / \tan \delta$, $x=1+(\tan \delta)^{2}$, and $\tan \delta=G^{\prime \prime} / G^{\prime}$ is called the loss tangent. The relevant values of the two moduli are determined from a representation as in Fig. 1.

To estimate the strain rates in the crack propagation zone, we analyze the profiles in terms of the de Gennes viscoelastic trumpet model [24]. Based on the stress field for an infinitely sharp line crack, the overall shape of a fracture profile $u(x)$ is divided into different spatial regions 
(a)

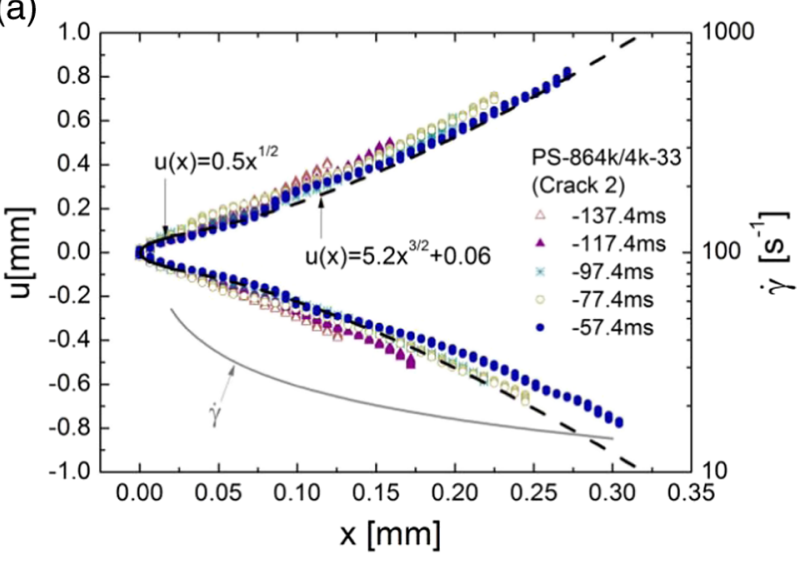

(b)
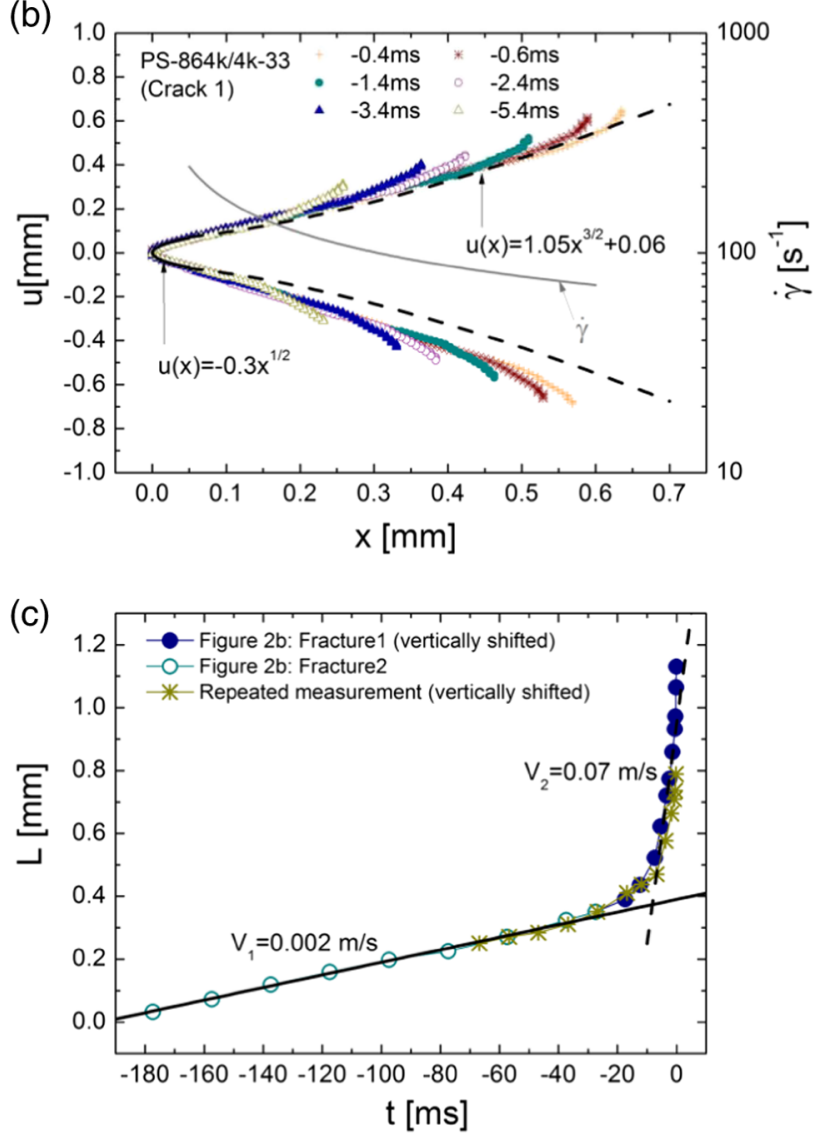

FIG. 4. Crack profile and crack length at different times during crack propagation for PS-864k/4k-33 stretched at $0.1 \mathrm{~s}^{-1}$ at $120^{\circ} \mathrm{C}$. (a) Corresponding to crack 2 in Fig. 3. (b) Corresponding to crack 1 in Fig. 3. The black solid lines are $u(x)=a x^{1 / 2}$, and the black dashed lines are $u(x)=b x^{3 / 2}+c$, where $a, b$, and $c$ are constants and have been adjusted to fit the captured profiles. The gray solid lines are the local shear rates. (c) Crack length as a function of time obtained from two independent measurements. Depending on the angle of the facture facing the camera, the actual crack length may be longer than observed. Therefore, the plots in the figure are vertically shifted to get a master curve. The shift of measurements when compared to the dashed lines visible in (b) may also be due to the slight angle mismatch between the crack and the camera. depending on the distance $x$ from the crack tip. According to the trumpet model, in region I where $x<V \tau$ ( $\tau$ is the smallest time constant), the viscous dissipation is negligible and the material can be considered as an elastic solid. A classical parabolic shape $u(x) \sim x^{1 / 2}$ is expected in this region. We use the Kuhn segment relaxation time $\tau=\tau_{0}$ from Fig. 1. In region II where $x>V \tau$, the material can be considered as a viscous liquid and the fracture shape is predicted as $u(x) \sim x^{3 / 2}$. Therefore, the overall fracture profile looks like a trumpet. In the work by Tabuteau et al. $[10,11]$, the parabolic shape in region I has been experimentally observed for a viscoelastic emulsion. Saulnier et al. [25] observed the trumpet profile in regions I and II for adhesive fracture between a polymer melt and a solid surface. However, for cohesive fractures, the complete trumpet profile including the viscous region II has so far eluded observation.

Figures 4(a) and 4(b) plot the fracture profile $u(x)$ for PS-864k/4k-33 from Fig. 3. The overall shape of the fracture as a function of time is well described by a trumpet profile. All extracted fracture profiles are fitted with a narrow region of $u(x)=a x^{1 / 2}$ near the crack tip, while the remaining profile is described by $u(x)=b x^{3 / 2}+c$, where $a, b$, and $c$ are constants (see also Figs. S4 and S5 in Supplemental Material [17]). While the single relaxation time model by de Gennes describes the profile qualitatively, it does not give a complete quantitative description. Thus, the size of the elastic region near the crack tip is predicted to be about $V \tau_{0} \approx 60 \mathrm{~nm}$, which is considerably smaller than the optical resolution and the parabolic distance used in Figs. 4(a) and 4(b). The local shear rate during crack propagation is calculated as $\dot{\gamma}=V\left(d^{2} u / d x^{2}\right)$, which is plotted in Figs. 4(a) and 4(b) for the viscous region $\left[u(x) \sim x^{3 / 2}\right]$ (see also Fig. S5 in Supplemental Material [17]). The shear rate in Fig. 4(a) corresponds to the slow stage $(V=2 \mathrm{~mm} / \mathrm{s})$ while in Fig. 4(b) it corresponds to the fast stage $(V=70 \mathrm{~mm} / \mathrm{s})$. It is clear that the frequencies (see Fig. 1) that correspond to the local shear rate in the crack propagation are in the range $1 / \tau_{e}<\omega<1 / \tau_{0}$ where the viscous component (energy dissipation) dominates the elastic component $\left(G^{\prime \prime}>G^{\prime}\right)$. This is consistent with the fact that most of the crack profile follows the viscous part of the trumpet model. On the other hand, the moduli in this regime predict a shear wave velocity much larger than the observed propagation speed $V$. Therefore, we expect that a full nonlinear simulation of the crack propagation will be needed to model the process.

In this Letter we have shown elastic fracture of model polymer liquids under well-defined extensional deformations. In all observed cases, the fracture initiates at the edge and propagates inward, thereby breaking the axis symmetry of the liquid filament. The crack profiles are qualitatively described by the de Gennes trumpet model with brittle conditions near the fracture tip and a viscous region in a tearing zone away from the tip. The highly reproducible 
fracture conditions as well as the simultaneous independent initiation of more than one crack strongly support the argument ([13]) that thermally induced fluctuations are responsible for crack initiation in polymer melts or solutions. We further hypothesize that such fluctuations lead to spots of lower entanglement density in the sample, but a quantitative model is needed to prove the matter.

Financial support from the Aage og Johanne LouisHansen Foundation is gratefully acknowledged. We thank Professor Suzanne M. Fielding from Durham University, Professor Dimitris Vlassopoulos from IESL-FORTH, and Professor Christian F. Niordson from DTU Mechanical Engineering for instructive comments.

*oh@kt.dtu.dk

[1] A. Ya. Malkin and C. J. S. Petrie, Some conditions for rupture of polymer liquids in extension, J. Rheol. 41, 1 (1997).

[2] S. M. Fielding, Criterion for Extensional Necking Instability in Polymeric Fluids, Phys. Rev. Lett. 107, 258301 (2011).

[3] Y. Ide and J. White, Investigation of failure during elongational flow of polymer melts, J. Non-Newtonian Fluid Mech. 2, 281 (1977).

[4] D. M. Hoyle and S. M. Fielding, Age-dependent modes of extensional necking instability in soft glassy materials, Phys. Rev. Lett. 114, 158301 (2015).

[5] A. R. C. Baijon and M. O. Robbins, Energy dissipation during rupture of adhesive bonds, Science 271, 482 (1996).

[6] D. Bonn, H. Kellay, M. Prochnow, K. Ben-Djemiaa, and J. Meunier, Delayed fracture of an inhomogeneous soft solid, Science 280, 265 (1998).

[7] C. Hui, T. Tang, Y. Lin, and M. K. Chaudhury, Failure of elastomeric polymers due to rate dependent bond rupture, Langmuir 20, 6052 (2004).

[8] E. Ducrot, Y. Chen, M. Bulters, R. P. Sijbesma, and C. Creton, Toughening elastomers with sacrificial bonds and watching them break, Science 344, 186 (2014).

[9] R. Longa and C. Hui, Crack tip fields in soft elastic solids subjected to large quasistatic deformation-a review, Extreme Mechanics Letters 4, 131 (2015).
[10] H. Tabuteau, S. Mora, G. Porte, M. Abkarian, and C. Ligoure, Microscopic mechanisms of the brittleness of viscoelastic fluids, Phys. Rev. Lett. 102, 155501 (2009).

[11] H. Tabuteau, S. Mora, M. Ciccotti, C. Huid, and C. Ligoure, Propagation of a brittle fracture in a viscoelastic fluid, Soft Matter 7, 9474 (2011).

[12] A. A. Griffith, The phenomena of rupture and flow in solids, Phil. Trans. R. Soc. A 221, 163 (1921).

[13] Y. Pomeau, Brisure spontanée de cristaux bidimensionnels courbés, C. R. Acad. Sci. Paris 314 II, 553 (1992).

[14] Q. Huang, L. Hengeller, N. J. Alvarez, and O. Hassager, Bridging the gap between polymer melts and solutions in extensional rheology, Macromolecules 48, 4158 (2015).

[15] S. L. Wingstrand, N. J. Alvarez, Q. Huang, and O. Hassager, Linear and nonlinear universality in the rheology of polymer melts and solutions, Phys. Rev. Lett. 115, 078302 (2015).

[16] M. Doi and S. F. Edwards, The Theory of Polymer Dynamics (Oxford University Press, New York, 1986).

[17] See Supplemental Material at http://link.aps.org/ supplemental/10.1103/PhysRevLett.117.087801 for material properties and experimental methods, which includes Ref. [18].

[18] J. Fang, M. Kröger, and H. C. Öttinger, A thermodynamically admissible reptation model for fast flows of entangled polymers. II. Model predictions for shear and extensional flows, J. Rheol. 44, 1293 (2000).

[19] M. Rubinstein and R. H. Colby, Polymer Physics (Oxford University Press, New York, 2003).

[20] Q. Huang, M. Mangnus, N. J. Alvarez, R. Koopmans, and O. Hassager, A new look at extensional rheology of low-densitypolyethylene, Rheol. Acta 55, 343 (2016).

[21] J. M. Román Marín, J. K. Huusom, N. J. Alvarez, Q. Huang, H. K. Rasmussen, A. Bach, A. L. Skov, and O. Hassager, A control scheme for filament stretching rheometers with application to polymer melts, J. Non-Newtonian Fluid Mech. 194, 14 (2013).

[22] R. B. Bird, R. C. Armstrong, and O. Hassager, Dynamics of Polymeric Liquids (Wiley, New York, 1987).

[23] C. Ligoure and S. Mora, Fractures in complex fluids: the case of transient networks, Rheol. Acta 52, 91 (2013).

[24] P. G. de Gennes, Soft adhesives, Langmuir 12, 4497 (1996).

[25] F. Saulnier, T. Ondarcuhu, A. Aradian, and E. Raphael, Adhesion between a viscoelastic material and a solid surface, Macromolecules 37, 1067 (2004). 